respectively. Approximately $70-75 \%$ of at-risk workers were probably or possibly exposed to any of the pesticides. Glyphosate exposure was most common among workers in oilseed (29\%) and dry pea/bean farms (28\%), along with those providing support activities for farms (31\%). 2,4-D exposure was most common in corn (28\%), other grain $(28 \%)$, and soybean farms (27\%), while chlorothalonil exposure was more likely among greenhouse, nursery and floriculture workers (42\%) and those working on farms (28\%, for occupations not captured by the CEAG). Regional variations reflected differences in farm types by province.

Conclusion This study estimated the prevalence of occupational pesticide exposure in Canada, and findings can support priority setting for future research and data collection.

\section{P-113 AN EXPLORATION OF DISPENSING ERRORS AMONG THE HOSPITAL PHARMACISTS IN TAIWAN}

${ }^{1}$ Kuan-Han Lin, Yun-Chia Chang. ${ }^{~}$ Asia University, Taiwan

\subsection{6/OEM-2021-EPI.202}

Introduction Dispensing errors are among the most common medical errors. Previous studies found that environmental factors, educational training, physical and mental health were factors affecting dispensing errors among pharmacists. However, the associations among burnout, sleep quality and dispensing error in pharmacists are still unknown.

Objectives The aims of this study were to investigate the frequency of dispensing errors among the hospital pharmacists in Taiwan and to determine the risk factors associated with it.

Methods This was a cross-sectional study. The electronic questionnaires were sent to the pharmacists from multiple centers. The questionnaire included demographic characteristics, the frequencies of dispensing errors in the past three months, environmental factors, Chinese version of Copenhagen Burnout Inventory, Chinese version of Pittsburgh Sleep Quality Index, and continuing education questions. Logistic regression models were used to assess the association between environmental factors, burnout, sleep quality, continuing education and dispensing errors.

Results A total of 370 pharmacists completed the questionnaire. Among them, 96.5\% reported dispensing errors in the past three months. In the univariate logistic analysis, pharmacists experienced dispensing errors were more likely to have shorter working years (Odds Ratio $(\mathrm{OR})=0.91$, 95\% Confidence Interval (CI) : 0.85-0.98), shift work (OR=4.75, 95\% CI : 1.51-14.89), shorter working hour per day $(\mathrm{OR}=0.80$, 95\% CI: 0.64-0.99), lower personal burnout score $(\mathrm{OR}=$ 0.97, 95\% CI: 0.94-0.99) and lower over-commitment score $(\mathrm{OR}=0.96$, 95\% CI: 0.94-0.99) compared with those without dispensing errors. The multivariate logistic regression model indicated that after adjusting for all possible risk factors, shorter working years (adjusted Odds Ratio $(\mathrm{aOR})=$ 0.92, 95\% CI: 0.85-0.99) and shift work (aOR $=4.63,95 \%$ CI: 1.39-15.45) was associated with dispensing errors among pharmacists.

Conclusion A significant proportion of pharmacists experienced dispensing errors. Development of stress management programs to enhance pharmacists' physical and psychological wellbeing and to improve job performance is warranted.

\section{P-115 PRO-MENTA: IMPACT OF MAJOR ORGANIZATIONAL CHANGES ON EMPLOYEE MENTAL HEALTH AND WORKPLACE PRODUCTIVITY}

'Lea Nørgaard Sørensen, Morten Vejs Willert, Johan Høy Jensen, Vita Ligaya Dalgaard, Marianne Kyndi. 'Aarhus University Hospital, Denmark

\subsection{6/OEM-2021-EPI.203}

Introduction The health care system of Denmark is currently undergoing major centralizations. Relocation of workplaces as well as mergers and split-ups may impact both health and productivity among employees and managers. The unification of all hospital units in Aarhus, Denmark, poses an opportunity to study the short- and long-term consequences of different degrees of organizational change during a fixed event.

Objectives The primary aims are to: 1) investigate the consequences of major organizational changes on absenteeism and mental health of employees and workplace productivity and staff turnover, and 2) identify risk factors for negative consequences on mental health, sickness absence, productivity, and staff turnover, in order to identify risk groups for targeted prevention.

Methods Using national and regional registers we will establish a cohort of 10,000 individuals employed at Aarhus University Hospital in the period from 2011-2020. Hospital units moving at different time points through 2016-2019 will be aligned on the index date defined by their relocation and followed up to 5 years after and prior to this.

Results Based on the life event literature we expect to find a delayed effect of major organisational change on sickness absence and mental health, and an immediate effect on staff turnover and productivity. We hypothesize these effects as more pronounced for units which in addition to relocating also merge/split, representing greater organisational change. We also expect to identify risk factors for the chosen outcomes based on job titles, age, seniority, and work unit characteristics such as number of employees, patient centred vs. service/ administrative, and out-patient vs. in-patient wards.

Conclusion This project is expected to produce valuable knowledge about the mental health and/or economic consequences of major organizational changes in the health care system. This can inform preventive actions targeting specific employee groups in future centralization projects.

\section{P-118 MANAGEMENT OF CAUSAL UNCERTAINTY : CAN A SICK BUILDING SYNDROM BECOME CHRONIC ?}

${ }^{1}$ Christine Meffre, Yoann Dominique, Juliette Chatelot, Eve Bourgkard, Michel Vernay. ${ }^{1}$ Agence Nationale de Santé Publique, France

\subsection{6/OEM-2021-EPI.204}

Objectives In 2014, the head of a elderly care facility (ECF) alerted the regional health authority for chronic irritative symptoms among office workers. Symptoms started in 2009 for 4 workers back in a newly rehabilitated building. From 2009 to 2014, the first analysis of indoor air quality lead to cleaning and disinfection of the air treatment plant. Furthermore no toxic compounds were identified in the materials used. In 2014, out of 21 office workers, 13 declared irritative symptoms, and the French national public health agency was called to carry out epidemiological investigations. 